\title{
Study of Fixed Point Theorems for Higher Dimension in Partially Ordered Metric Spaces
}

\author{
Ibtisam Masmali, Sumitra Dalal \\ College of Science, Jazan University, Jazan, K.S.A \\ Email: ibtisam234@hotmail.com, mathsqueen_d@yahoo.com
}

Received 5 January 2016; accepted 15 March 2016; published 18 March 2016

Copyright $@ 2016$ by authors and Scientific Research Publishing Inc.

This work is licensed under the Creative Commons Attribution International License (CC BY). http://creativecommons.org/licenses/by/4.0/

c) (i) Open Access

\begin{abstract}
In this paper, we establish the existence and uniqueness of fixed points of operator $F: X^{n} \rightarrow X$, when $n$ is an arbitrary positive integer and $X$ is a partially ordered complete metric space. We have shown examples to verify our work. Our results generalize the recent fixed point theorems cited in [1]-[4] etc. and include several recent developments.
\end{abstract}

\section{Keywords}

n-Tupled Coincidence Points, n-Tupled Coincidence Fixed Points, Compatible Maps, Fixed Points and Partially Ordered Metric Spaces

\section{Introduction}

The metric fixed point theory plays a vital role to solve the problems related to variational inequalities, optimization, approximation theory, etc. Many authors (for detail, see [1]-[10]) have discussed fixed point results in partially ordered metric spaces. In particular, Bhaskar and Lakshmikantham [3], Nieto and Rodriguez-Lopez [11], Agarwal et al. [12] and Ran and Recuring [13] proved some new results for contractions in partially ordered metric spaces.

Bhaskar and Lakshmikantham [3] proposed the study of a coupled fixed point in ordered metric spaces and as an application they proved the existence and uniqueness of solutions for a periodic boundary value problem. Nguyen et al. [14], Berinde and Borcut [15] and Karpinar [8] introduced tripled and quadruple fixed point theorems as a generalization and extension of the coupled fixed point theorem. For comprehensive description of such work, we refer to [16]-[21]. Very recently, Imdad et al. [22] have introduced the concept of n-tupled coincidence point and proved n-tupled coincidence point results for commuting maps in metric spaces. Motivated by 
the work of M. Imdad, we introduce the notion of compatibility for n-tupled coincidence points and prove n-tupled coincidence point and n-tupled fixed point for compatible maps satisfying different contractive conditions in partially ordered metric spaces.

Jungck [1] obtained common fixed point results for commuting maps in metric spaces. The concept of commuting maps has been generalized in various directions over the years. One such generalization which is weaker than commuting is the concept of compatibility introduced by Jungck [23].

\section{Prilimaries}

Definition 2.1 [4] Let $(X, \preceq)$ be a partially ordered set equipped with a metric $d$ such that $(X, d)$ is a metric space. Further, equip the product space $X \times X$ with the following partial ordering:

For $(x, y),(u, v) \in X \times X$, define $(u, v) \preceq(x, y) \Leftrightarrow x \succeq u, y \preceq v$.

Definition 2.2 [4] Let $(X, \preceq)$ be a partially ordered set and $F: X \times X \rightarrow X$ then $F$ enjoys the mixed monotone property if $F(x, y)$ is monotonically non-decreasing in $x$ and monotonically non-increasing in $y$, that is, for any $x, y \in X$,

$$
x_{1}, x_{2} \in X, x_{1} \preceq x_{2} \Rightarrow F\left(x_{1}, y\right) \preceq F\left(x_{2}, y\right) \text { and } y_{1}, y_{2} \in X, y_{1} \preceq y_{2} \Rightarrow F\left(x, y_{1}\right) \succeq F\left(x, y_{2}\right) \text {. }
$$

Definition 2.3 [4] Let $(X, \preceq)$ be a partially ordered set and $F: X \times X \rightarrow X$, then $(x, y) \in X \times X$ is called a coupled fixed point of the mapping $F$ if $F(x, y)=x$ and $F(y, x)=y$.

Definition 2.4 [4] Let $(X, \preceq)$ be a partially ordered set and $F: X \times X \rightarrow X$ and $g: X \rightarrow X$ then $F$ enjoys the mixed g-monotone property if $F(x, y)$ is monotonically g-non-decreasing in $x$ and monotonically gnon-increasing in $y$, that is, for any $x, y \in X$,

$$
\begin{aligned}
& x_{1}, x_{2} \in X, g\left(x_{1}\right) \preceq g\left(x_{2}\right) \Rightarrow F\left(x_{1}, y\right) \preceq F\left(x_{2}, y\right), \text { for any } y \in X, \\
& y_{1}, y_{2} \in X, g\left(y_{1}\right) \preceq g\left(y_{2}\right) \Rightarrow F\left(x, y_{1}\right) \succeq F\left(x, y_{2}\right), \text { for any } x \in X .
\end{aligned}
$$

Definition 2.5 [4] Let $(X, \preceq)$ be a partially ordered set and $F: X \times X \rightarrow X$ and $g: X \rightarrow X$, then $(x, y) \in X \times X$ is called a coupled coincidence point of the maps $F$ and $g$ if $F(x, y)=g x$ and $F(y, x)=g y$.

Definition 2.6 [4] Let $(X, \preceq)$ be a partially ordered set, then $(x, y) \in X \times X$ is called a coupled fixed point of the maps $F: X \times X \rightarrow X$ and $g: X \rightarrow X$ if $g x=F(x, y)=x$ and $g y=F(y, x)=y$.

\section{Main Results}

Imdad et al. [22] introduced the concept of n-tupled fixed point and n-tupled coincidence point given by considering $n$ to be an even integer but throughout, we will consider $n$, a positive integer, in this paper.

Definition 2.7 Let $(X, \preceq)$ be a partially ordered set and $F: \prod_{i=1}^{r} X^{i} \rightarrow X$ then $F$ is said to have the mixed monotone property if $F$ is non-decreasing in its odd position arguments and non-increasing in its even positions arguments, that is, if,

1) For all $x_{1}^{1}, x_{2}^{1} \in X, x_{1}^{1} \preceq x_{2}^{1} \Rightarrow F\left(x_{1}^{1}, x^{2}, x^{3}, \cdots, x^{r}\right) \preceq F\left(x_{2}^{1}, x^{2}, x^{3}, \cdots, x^{r}\right)$,

2) For all $x_{1}^{2}, x_{2}^{2} \in X, x_{1}^{2} \preceq x_{2}^{2} \Rightarrow F\left(x^{1}, x_{1}^{2}, x^{3}, \cdots, x^{r}\right) \succeq F\left(x^{1}, x_{2}^{2}, x^{3}, \cdots, x^{r}\right)$,

3) For all $x_{1}^{3}, x_{2}^{3} \in X, x_{1}^{3} \preceq x_{2}^{3} \Rightarrow F\left(x^{1}, x^{2}, x_{1}^{3}, x^{4}, \cdots, x^{r}\right) \preceq F\left(x^{1}, x^{2}, x_{2}^{3}, x^{4}, \cdots, x^{r}\right)$,

For all $x_{1}^{r}, x_{2}^{r} \in X, x_{1}^{r} \preceq x_{2}^{r} \Rightarrow F\left(x^{1}, x^{2}, x^{3}, \cdots, x_{1}^{r}\right) \preceq F\left(x^{1}, x^{2}, x^{3}, \cdots, x_{2}^{r}\right) \quad$ (if $r$ is odd),

For all $x_{1}^{r}, x_{2}^{r} \in X, x_{1}^{r} \preceq x_{2}^{r} \Rightarrow F\left(x^{1}, x^{2}, x^{3}, \cdots, x_{1}^{r}\right) \succeq F\left(x^{1}, x^{2}, x^{3}, \cdots, x_{2}^{r}\right)$ (if $r$ is even).

Definition 2.8 Let $(X, \preceq)$ be a partially ordered set and $F: \prod_{i=1}^{r} X^{i} \rightarrow X$ and $g: X \rightarrow X$ be two maps.

Then $F$ is said to have the mixed g-monotone property if $F$ is g-non-decreasing in its odd position arguments and g-non-increasing in its even positions arguments, that is, if, 
1) For all $x_{1}^{1}, x_{2}^{1} \in X, g x_{1}^{1} \preceq g x_{2}^{1} \Rightarrow F\left(x_{1}^{1}, x^{2}, x^{3}, \cdots, x^{r}\right) \preceq F\left(x_{2}^{1}, x^{2}, x^{3}, \cdots, x^{r}\right)$,

2) For all $x_{1}^{2}, x_{2}^{2} \in X, g x_{1}^{2} \preceq g x_{2}^{2} \Rightarrow F\left(, x^{1}, x_{1}^{2}, x^{3}, \cdots, x^{r}\right) \succeq F\left(x^{1}, x_{2}^{2}, x^{3}, \cdots, x^{r}\right)$,

3) For all $x_{1}^{3}, x_{2}^{3} \in X, g x_{1}^{3} \preceq g x_{2}^{3} \Rightarrow F\left(x^{1}, x^{2}, x_{1}^{3}, \cdots, x^{r}\right) \preceq F\left(x^{1}, x^{2}, x_{2}^{3}, \cdots, x^{r}\right)$,

For all $x_{1}^{r}, x_{2}^{r} \in X, g x_{1}^{r} \preceq g x_{2}^{r} \Rightarrow F\left(x^{1}, x^{2}, x^{3}, \cdots, x_{1}^{r}\right) \preceq F\left(x^{1}, x^{2}, \cdots, x_{2}^{r}\right)$, (if $r$ is odd),

For all $x_{1}^{r}, x_{2}^{r} \in X, g x_{1}^{r} \preceq g x_{2}^{r} \Rightarrow F\left(x^{1}, x^{2}, x^{3}, \cdots, x_{1}^{r}\right) \succeq F\left(x^{1}, x^{2}, \cdots, x_{2}^{r}\right)$, (if $r$ is even).

Definition 2.9 [22] Let $X$ be a nonempty set. An element $\left(x^{1}, x^{2}, x^{3}, \cdots, x^{r}\right) \in \prod_{i=1}^{r} X^{i}$ is called an r-tupled fixed point of the mapping $F: \prod_{i=1}^{r} X^{i} \rightarrow X$ if

$$
\begin{aligned}
x^{1} & =F\left(x^{1}, x^{2}, x^{3}, \cdots, x^{r}\right), \\
x^{2} & =F\left(x^{2}, x^{3}, \cdots, x^{r}, x^{1}\right), \\
& \vdots \\
x^{r} & =F\left(x^{r}, x^{1}, x^{2}, \cdots, x^{r-1}\right) .
\end{aligned}
$$

Example 1. Let $(R, d)$ be a partial ordered metric space under natural setting and let $F: \prod_{i=1}^{r} X^{i} \rightarrow X$ be mapping defined by

$$
F\left(x^{1}, x^{2}, x^{3}, \cdots, x^{r}\right)=\sin \left(x^{1} \cdot x^{2} \cdot x^{3} \cdots x^{r}\right), \text { for any } x^{1}, x^{2}, x^{3}, \cdots, x^{r} \in X,
$$

then $(0,0,0, \cdots, 0)$ is an r-tupled fixed point of $F$.

Definition 2.10 [22] Let $X$ be a nonempty set. An element $\left(x^{1}, x^{2}, x^{3}, \cdots, x^{r}\right) \in \prod_{i=1}^{r} X^{i}$ is called an r-tupled coincidence point of the maps $F: \prod_{i=1}^{r} X^{i} \rightarrow X$ and $g: X \rightarrow X$ if

$$
\begin{aligned}
g x^{1} & =F\left(x^{1}, x^{2}, x^{3}, x^{r}\right), \\
g x^{2} & =F\left(x^{2}, x^{3}, \cdots, x^{r}, x^{1}\right), \\
g x^{3} & =F\left(x^{3}, \cdots, x^{r}, x^{1}, x^{2}\right), \\
& \vdots \\
g x^{r} & =F\left(x^{r}, x^{2}, x^{3}, \cdots, x^{r-1}\right) .
\end{aligned}
$$

Example 2. Let $(R, d)$ be a partial ordered metric space under natural setting and let $F: \prod_{i=1}^{r} X^{i} \rightarrow X$ and $g: X \rightarrow X$ be maps defined by

$$
F\left(x^{1}, x^{2}, x^{3}, \cdots, x^{r}\right)=\sin x^{1} \cdot \cos x^{2} \cdot \sin x^{3} \cdot \cos x^{4} \cdots \sin x^{r-1} \cdot \cos x^{r}, g(x)=\sin x,
$$

for any $x^{1}, x^{2}, x^{3}, \cdots, x^{r} \in X$, then $\left\{\left(x^{1}, x^{2}, x^{3}, \cdots, x^{r}\right), x^{i}=m \pi, m \in N, 1 \leq i \leq r\right\}$ is an r-tupled coincidence point of $F$ and $g$.

Definition 2.11 [22] Let $X$ be a nonempty set. An element $\left(x^{1}, x^{2}, x^{3}, \cdots, x^{r}\right) \in \prod_{i=1}^{r} X^{i}$ is called an r-tupled fixed point of the maps $F: \prod_{i=1}^{r} X^{i} \rightarrow X$ and $g: X \rightarrow X$ if

$$
\begin{gathered}
x^{1}=g x^{1}=F\left(x^{1}, x^{2}, x^{3}, \cdots, x^{r}\right), \\
x^{2}=g x^{2}=F\left(x^{2}, x^{3}, \cdots, x^{r}, x^{1}\right), \\
x^{3}=g x^{3}=F\left(x^{3}, \cdots, x^{r}, x^{1}, x^{2}\right), \\
\vdots \\
x^{r}=g x^{r}=F\left(x^{r}, x^{1}, x^{2}, \cdots, x^{r-1}\right) .
\end{gathered}
$$


Now, we define the concept of compatible maps for r-tupled maps.

Definition 2.12 Let $(X, \preceq)$ be a partially ordered set, then the maps $F: \prod_{i=1}^{r} X^{i} \rightarrow X$ and $g: X \rightarrow X$ are called compatible if

$$
\begin{gathered}
\lim _{n \rightarrow \infty}\left(g\left(F\left(x_{n}^{1}, x_{n}^{2}, \cdots, x_{n}^{r}\right)\right), F\left(g x_{n}^{1}, g x_{n}^{2}, \cdots, g x_{n}^{r}\right)\right)=0, \\
\lim _{n \rightarrow \infty}\left(g\left(F\left(x_{n}^{2}, x_{n}^{3}, \cdots, x_{n}^{r}, x_{n}^{1}\right)\right), F\left(g x_{n}^{2}, g x_{n}^{3}, \cdots, g x_{n}^{r}, g x_{n}^{1}\right)\right)=0, \\
\lim _{n \rightarrow \infty}\left(g\left(F\left(x_{n}^{3}, \cdots, x_{n}^{r}, x_{n}^{1}, x_{n}^{2},\right)\right), F\left(g x_{n}^{3}, \cdots, g x_{n}^{r}, g x_{n}^{1}, g x_{n}^{2}\right)\right)=0, \\
\vdots \\
\lim _{n \rightarrow \infty}\left(g\left(F\left(x_{n}^{r}, x_{n}^{1}, x_{n}^{2} \cdots, x_{n}^{r-1}\right)\right), F\left(g x_{n}^{r}, g x_{n}^{1}, g x_{n}^{2}, \cdots, g x_{n}^{r-1}\right)\right)=0,
\end{gathered}
$$

whenever, $\left\{x_{n}^{1}\right\},\left\{x_{n}^{2}\right\},\left\{x_{n}^{3}\right\}, \cdots,\left\{x_{n}^{r}\right\}$ are sequences in $X$ such that

$$
\begin{gathered}
\lim _{n \rightarrow \infty} F\left(x_{n}^{1}, x_{n}^{2}, x_{n}^{3}, \cdots, x_{n}^{r}\right)=\lim _{n \rightarrow \infty} g\left(x_{n}^{1}\right)=x^{1}, \\
\lim _{n \rightarrow \infty} F\left(x_{n}^{2}, x_{n}^{3}, \cdots, x_{n}^{r}, x_{n}^{1}\right)=\lim _{n \rightarrow \infty} g\left(x_{n}^{2}\right)=x^{2}, \\
\lim _{n \rightarrow \infty} F\left(x_{n}^{3}, x_{n}^{4}, \cdots, x_{n}^{1}, x_{n}^{2}\right)=\lim _{n \rightarrow \infty} g\left(x_{n}^{3}\right)=x^{3}, \\
\vdots \\
\lim _{n \rightarrow \infty} F\left(x_{n}^{r}, x_{n}^{1}, x_{n}^{2}, \cdots, x_{n}^{r-1}\right)=\lim _{n \rightarrow \infty} g\left(x_{n}^{r}\right)=x^{r} .
\end{gathered}
$$

For some $x^{1}, x^{2}, x^{3}, \cdots, x^{r} \in X$.

Imdad et al. [22] proved the following theorem:

Theorem 3.1 Let $(X, \preceq)$ be a partially ordered set equipped with a metric $\mathrm{d}$ such that $(X, d)$ is a complete metric space. Assume that there is a function $\varphi:[0, \infty) \rightarrow[0, \infty)$ with $\varphi(t)<t$ and $\lim _{r \rightarrow t^{+}} \varphi(r)<t$ for each $t>0$. Further, let $F: \prod_{i=1}^{r} X^{i} \rightarrow X$ and $g: X \rightarrow X$ be two maps such that $F$ has the mixed g-monotone property satisfying the following conditions:

(i) $F\left(\prod_{i=1}^{r} X^{i}\right) \subseteq g(X)$,

(ii) $\mathrm{g}$ is continuous and monotonically increasing,

(iii) the pair $(g, F)$ is commuting,

(iv) $d\left(F\left(x^{1}, x^{2}, x^{3}, \cdots, x^{r}\right), F\left(y^{1}, y^{2}, y^{3}, \cdots, y^{r}\right)\right) \leq \varphi\left(\frac{1}{r} \sum_{n=1}^{r} d\left(g\left(x^{n}\right), g\left(y^{n}\right)\right)\right)$ for all $x^{1}, x^{2}, x^{3}, \cdots, x^{r}, y^{1}, y^{2}, y^{3}, \cdots, y^{r} \in X$, with $g x^{1} \preceq g y^{1}, g x^{2} \succeq g y^{2}, g x^{3} \preceq g y^{3}, \cdots, g x^{r} \succeq g y^{r}$ if $r$ is even and $g x^{r} \preceq g y^{r}$ if $r$ is odd. Also, suppose that either

a) $F$ is continuous or

b) $X$ has the following properties:

(i) If a non-decreasing sequence $\left\{x_{n}\right\} \rightarrow x$ then $x_{n} \leq x$ for all $n \geq 0$.

(ii) If a non-increasing sequence $\left\{y_{n}\right\} \rightarrow y$ then $y \leq y_{n}$ for all $n \geq 0$.

If there exist $x_{0}^{1}, x_{0}^{2}, x_{0}^{3}, \cdots, x_{0}^{r} \in X$ such that

(iv) $g x_{0}^{1} \preceq F\left(x_{0}^{1}, x_{0}^{2}, x_{0}^{3}, x_{0}^{r}\right)$,

$$
\begin{aligned}
& g x_{0}^{2} \succeq F\left(x_{0}^{2}, x_{0}^{3}, \cdots, x_{0}^{r}, x_{0}^{1}\right), \\
& g x_{0}^{3} \succeq F\left(x_{0}^{3}, \cdots, x_{0}^{r}, x_{0}^{1}, x_{0}^{2}\right),
\end{aligned}
$$


$g x_{0}^{r} \preceq F\left(x_{0}^{r}, x_{0}^{1}, x_{0}^{2}, x_{0}^{3}, \cdots, x_{0}^{r-1}\right), \quad$ if $r$ is odd,

$g x_{0}^{r} \succeq F\left(x_{0}^{r}, x_{0}^{1}, x_{0}^{2}, x_{0}^{3}, \cdots, x_{0}^{r-1}\right)$, if $r$ is even.

Then $F$ and $g$ have a r-tupled coincidence point, i.e. there exist $x^{1}, x^{2}, x^{3}, \cdots, x^{r} \in X$ such that

$$
\begin{aligned}
g x^{1} & =F\left(x^{1}, x^{2}, x^{3}, \cdots, x^{r}\right), \\
g x^{2} & =F\left(x^{2}, x^{3}, \cdots, x^{r}, x^{1}\right), \\
g x^{3} & =F\left(x^{3}, \cdots, x^{r}, x^{1}, x^{2}\right), \\
& \vdots \\
g x^{r} & =F\left(x^{r}, x^{1}, x^{2}, x^{3}, \cdots, x^{r-1}\right) .
\end{aligned}
$$

Now, we prove our main result as follows:

Theorem 3.2 Let $(X, \preceq)$ be a partially ordered set equipped with a metric $d$ such that $(X, d)$ is a complete metric space. Assume that there is a function $\varphi:[0, \infty) \rightarrow[0, \infty)$ with $\varphi(t)<t$ and $\lim _{r \rightarrow t^{+}} \varphi(r)<t$ for each $t>0$. Further let $F: \prod_{i=1}^{r} X^{i} \rightarrow X$ and $g: X \rightarrow X$ be two maps such that $F$ has the mixed g-monotone property satisfying the following conditions:

(3.1) $F\left(\prod_{i=1}^{r} X^{i}\right) \subseteq g(X)$,

(3.2) $g$ is continuous and monotonically increasing,

(3.3) the pair $(g, F)$ is compatible,

(3.4) $d\left(F\left(x^{1}, x^{2}, x^{3}, \cdots, x^{r}\right), F\left(y^{1}, y^{2}, y^{3}, \cdots, y^{r}\right)\right) \leq \varphi\left(\max \left\{d\left(g\left(x^{n}\right), g\left(y^{n}\right)\right)\right\}\right)$,

For all $x^{1}, x^{2}, x^{3}, \cdots, x^{r}, y^{1}, y^{2}, y^{3}, \cdots, y^{r} \in X, n=1,2, \cdots, r$, with $g x^{1} \preceq g y^{1}, g x^{2} \succeq g y^{2}$, $g x^{3} \preceq g y^{3}, \cdots, g x^{r} \succeq g y^{r}$, if $r$ is even and $g x^{r} \preceq g y^{r}$, if $r$ is odd. Also, suppose that either

a) $F$ is continuous or

b) $X$ has the following properties:

(i) If a non-decreasing sequence $\left\{x_{n}\right\} \rightarrow x$ then $x_{n} \preceq x$ for all $n \geq 0$.

(ii) If a non-increasing sequence $\left\{y_{n}\right\} \rightarrow y$ then $y \preceq y_{n}$ for all $n \geq 0$.

If there exist $x_{0}^{1}, x_{0}^{2}, x_{0}^{3}, \cdots, x_{0}^{r} \in X$ such that

$$
\begin{aligned}
g x_{0}^{1} & \preceq F\left(x_{0}^{1}, x_{0}^{2}, x_{0}^{3}, \cdots, x_{0}^{r}\right), \\
g x_{0}^{2} & \succeq F\left(x_{0}^{2}, x_{0}^{3}, \cdots, x_{0}^{r}, x_{0}^{1}\right), \\
g x_{0}^{3} & \preceq F\left(x_{0}^{3}, \cdots, x_{0}^{r}, x_{0}^{1}, x_{0}^{2}\right), \\
& \vdots \\
g x_{0}^{r} & \succeq F\left(x_{0}^{r}, x_{0}^{1}, x_{0}^{2}, x_{0}^{3}, \cdots, x_{0}^{r-1}\right), \text { if } r \text { is even, } \\
g x_{0}^{r} & \preceq F\left(x_{0}^{r}, x_{0}^{1}, x_{0}^{2}, x_{0}^{3}, \cdots, x_{0}^{r-1}\right), \text { if } r \text { is odd. }
\end{aligned}
$$

Then $F$ and $g$ have a r-tupled coincidence point, i.e. there exist $x^{1}, x^{2}, x^{3}, \cdots, x^{r} \in X$ such that

$$
\begin{aligned}
g x^{1} & =F\left(x^{1}, x^{2}, x^{3}, \cdots, x^{r}\right), \\
g x^{2} & =F\left(x^{2}, x^{3}, \cdots, x^{r}, x^{1}\right), \\
g x^{3} & =F\left(x^{3}, \cdots, x^{r}, x^{1}, x^{2}\right), \\
& \vdots \\
g x^{r} & =F\left(x^{r}, x^{1}, x^{2}, x^{3}, \cdots, x^{r-1}\right) .
\end{aligned}
$$


Proof. Starting with $x_{0}^{1}, x_{0}^{2}, x_{0}^{3}, \cdots, x_{0}^{r} \in X$, we define the sequences $\left\{x_{n}^{1}\right\},\left\{x_{n}^{2}\right\},\left\{x_{n}^{3}\right\}, \cdots,\left\{x_{n}^{r}\right\}$ in $X$ as follows:

$$
\begin{aligned}
g x_{n+1}^{1} & =F\left(x_{n}^{1}, x_{n}^{2}, x_{n}^{1}, \cdots, x_{n}^{r}\right), \\
g x_{n+1}^{2} & =F\left(x_{n}^{2}, x_{n}^{3}, \cdots, x_{n}^{r}, x_{n}^{1}\right), \\
g x_{n+1}^{3} & =F\left(x_{n}^{3}, \cdots, x_{n}^{r}, x_{n}^{1}, x_{n}^{2}\right), \\
& \vdots \\
g x_{n+1}^{r} & =F\left(x_{n}^{r}, x_{n}^{1}, x_{n}^{2}, x_{n}^{3}, \cdots, x_{n}^{r-1}\right) .
\end{aligned}
$$

Now, we prove that for all $\mathrm{n} \geq 0$,

$$
g x_{n}^{1} \preceq g x_{n+1}^{1}, g x_{n}^{2} \succeq g x_{n+1}^{2}, g x_{n}^{3} \preceq g x_{n+1}^{3}, \cdots, g x_{n}^{r} \succeq g x_{n+1}^{r}, \text { if } r \text { is even and }
$$

$g x_{n}^{r} \preceq g x_{n+1}^{r}$, if $r$ is odd.

$$
\begin{gathered}
g x_{0}^{1} \preceq F\left(x_{0}^{1}, x_{0}^{2}, x_{0}^{3}, \cdots, x_{0}^{r}\right)=g x_{1}^{1}, \\
g x_{0}^{2} \succeq F\left(x_{0}^{2}, x_{0}^{3}, \cdots, x_{0}^{r}, x_{0}^{1}\right)=g x_{1}^{2}, \\
g x_{0}^{3} \preceq F\left(x_{0}^{3}, \cdots, x_{0}^{r}, x_{0}^{1}, x_{0}^{2}\right)=g x_{1}^{3},
\end{gathered}
$$

$\vdots$

$$
\begin{gathered}
g x_{0}^{r} \succeq F\left(x_{0}^{r}, x_{0}^{1}, x_{0}^{2}, x_{0}^{3}, \cdots, x_{0}^{r-1}\right)=g x_{1}^{r}, \text { if } r \text { is even, } \\
g x_{0}^{r} \preceq F\left(x_{0}^{r}, x_{0}^{1}, x_{0}^{2}, x_{0}^{3}, \cdots, x_{0}^{r-1}\right)=g x_{1}^{r}, \text { if } r \text { is old. }
\end{gathered}
$$

So (3.8) holds for $n=0$. Suppose that (3.8) holds for some $n>0$. Consider

$$
\begin{aligned}
& g x_{n+1}^{1}=F\left(x_{n}^{1}, x_{n}^{2}, x_{n}^{3}, \cdots, x_{n}^{r}\right) \\
& \preceq F\left(x_{n+1}^{1}, x_{n}^{2}, x_{n}^{3}, \cdots, x_{n}^{r}\right) \\
& \preceq F\left(x_{n+1}^{1}, x_{n+1}^{2}, x_{n}^{3}, \cdots, x_{n}^{r}\right) \\
& \preceq F\left(x_{n+1}^{1}, x_{n+1}^{2}, x_{n+1}^{3}, \cdots, x_{n}^{r}\right) \\
& \preceq F\left(x_{n+1}^{1}, x_{n+1}^{2}, \cdots, x_{n+1}^{r}\right)=g x_{n+2}^{1} \text {, } \\
& g x_{n+1}^{2}=F\left(x_{n}^{2}, x_{n}^{3}, \cdots, x_{n}^{r} x_{n}^{1}\right) \\
& \succeq F\left(x_{n+1}^{2}, x_{n}^{3}, \cdots, x_{n}^{r}, x_{n}^{1}\right) \\
& \succeq F\left(x_{n+1}^{2}, x_{n+1}^{3}, \cdots, x_{n}^{r}, x_{n}^{1}\right) \\
& \succeq F\left(x_{n+1}^{2}, x_{n+1}^{3}, \cdots, x_{n+1}^{r}, x_{n}^{1}\right) \\
& \succeq F\left(x_{n+1}^{1}, x_{n+1}^{2}, \cdots, x_{n+1}^{r}, x_{n+1}^{1}\right)=g x_{n+2}^{2}, \\
& g x_{n+1}^{3}=F\left(x_{n}^{3}, \cdots, x_{n}^{r}, x_{n}^{1}, x_{n}^{2},\right) \\
& \preceq F\left(x_{n+1}^{3}, \cdots, x_{n}^{r}, x_{n}^{1}, x_{n}^{2}\right) \\
& \preceq F\left(x_{n+1}^{3}, x_{n+1}^{4}, \cdots, x_{n}^{r}, x_{n}^{1}, x_{n}^{2}\right) \\
& \preceq F\left(x_{n+1}^{3}, x_{n+1}^{4}, \cdots, x_{n+1}^{r}, x_{n}^{1}, x_{n}^{2}\right) \\
& \preceq F\left(x_{n+1}^{3}, x_{n+1}^{4}, x_{n}^{5}, \cdots, x_{n+1}^{r}, x_{n+1}^{1}, x_{n}^{2}\right) \\
& \preceq F\left(x_{n+1}^{3}, x_{n+1}^{4}, \cdots, x_{n+1}^{r}, x_{n+1}^{1}, x_{n+1}^{2}\right)=g x_{n+2}^{3} \text {, }
\end{aligned}
$$




$$
\begin{aligned}
g x_{n+1}^{r} & =F\left(x_{n}^{r}, x_{n}^{1}, x_{n}^{2}, \cdots, x_{n}^{r-1}\right) \\
& \succeq F\left(x_{n+1}^{r}, x_{n}^{1}, x_{n}^{2}, \cdots, x_{n}^{r-1}\right) \\
& \succeq F\left(x_{n+1}^{r}, x_{n+1}^{1}, x_{n}^{2}, x_{n}^{3}, \cdots, x_{n}^{r-1}\right) \\
& \succeq F\left(x_{n+1}^{r}, x_{n+1}^{1}, x_{n+1}^{2}, \cdots, x_{n,}^{r-1}\right) \\
& \succeq F\left(x_{n+1}^{r}, x_{n+1}^{1}, x_{n}^{2}, \cdots, x_{n+1}^{r-1}\right)=g x_{n+2}^{r}, \text { if } r \text { is even, }
\end{aligned}
$$

and $g x_{n+1}^{r} \preceq F\left(x_{n+1}^{r}, x_{n+1}^{1}, x_{n}^{2}, \cdots, x_{n+1}^{r-1}\right)=g x_{n+2}^{r}$, if $r$ is odd.

Thus by induction (3.8) holds for all $n \geq 0$. Using (3.7) and (3.8)

$$
\begin{aligned}
d\left(g\left(x_{m}^{1}\right), g\left(x_{m+1}^{1}\right)\right) & =d\left(F\left(x_{m-1}^{1}, x_{m-1}^{2}, \cdots, x_{m-1}^{r}\right), F\left(x_{m}^{1}, x_{m}^{2}, \cdots, x_{m}^{r}\right)\right) \\
& \leq \varphi\left(\max d\left(g\left(x_{m-1}^{n}\right), g\left(x_{m}^{n}\right)\right)\right) .
\end{aligned}
$$

Similarly, we can inductively write

$$
d\left(g\left(x_{m}^{2}\right), g\left(x_{m+1}^{2}\right)\right) \leq \varphi\left(\max d\left(g\left(x_{m-1}^{n}\right), g\left(x_{m}^{n}\right)\right)\right),
$$

$\vdots$

$$
d\left(g\left(x_{m}^{r}\right), g\left(x_{m+1}^{r}\right)\right) \leq \varphi\left(\max d\left(g\left(x_{m-1}^{n}\right), g\left(x_{m}^{n}\right)\right)\right)
$$

Therefore, by putting

$$
\gamma_{m}=\max \left\{d\left(g\left(x_{m}^{1}\right), g\left(x_{m+1}^{1}\right)\right), d\left(g\left(x_{m}^{2}\right), g\left(x_{m+1}^{2}\right)\right), \cdots, d\left(g\left(x_{m}^{r}\right), g\left(x_{m+1}^{r}\right)\right)\right\} .
$$

We have,

$$
\begin{aligned}
\gamma_{m} & =\max \left\{d\left(g\left(x_{m}^{1}\right), g\left(x_{m+1}^{1}\right)\right), d\left(g\left(x_{m}^{2}\right), g\left(x_{m+1}^{2}\right)\right), \cdots, d\left(g\left(x_{m}^{r}\right), g\left(x_{m+1}^{r}\right)\right)\right\} \\
& \leq \varphi\left(\max \left\{d\left(g\left(x_{m-1}^{n}\right), g\left(x_{m}^{n}\right)\right)\right\}\right)=\varphi\left(\gamma_{m-1}\right) .
\end{aligned}
$$

Since $\varphi(t)<t$ for all $t>0$, therefore, $\gamma_{m} \leq \gamma_{m-1}$ for all $m$ so that $\left\{\gamma_{m}\right\}$ is a non-increasing sequence. Since it is bounded below, there is some $\gamma \geq 0$ such that

$$
\lim _{n \rightarrow \infty} \gamma_{n}=+\gamma .
$$

We shall show that $\gamma=0$. Suppose, if possible $\gamma>0$. Taking limit as $m \rightarrow \infty$ of both sides of (3.13) and keeping in mind our supposition that $\lim _{r \rightarrow t^{+}} \varphi(r)<t$ for all $t>0$, we have

$$
\gamma=\lim _{n \rightarrow \infty} \gamma_{m} \leq \varphi\left(\gamma_{m-1}\right)=\varphi(\gamma)<\gamma,
$$

this contradiction gives $\gamma=0$ and hence

$$
\lim _{n \rightarrow \infty}\left[\max \left\{d\left(g\left(x_{m}^{1}\right), g\left(x_{m+1}^{1}\right)\right), d\left(g\left(x_{m}^{2}\right), g\left(x_{m+1}^{2}\right)\right), \cdots, d\left(g\left(x_{m}^{r}\right), g\left(x_{m+1}^{r}\right)\right)\right\}\right]=0 .
$$

Next we show that all the sequences $\left\{g\left(x_{m}^{1}\right)\right\},\left\{g\left(x_{m}^{2}\right)\right\},\left\{g\left(x_{m}^{3}\right)\right\}, \cdots$, and $\left\{g\left(x_{m}^{r}\right)\right\}$ are Cauchy sequences. If possible, suppose that at least one of $\left\{g\left(x_{m}^{1}\right)\right\},\left\{g\left(x_{m}^{2}\right)\right\}, \cdots$, and $\left\{g\left(x_{m}^{r}\right)\right\}$ is not a Cauchy sequence. Then there 
exist $\epsilon>0$ and sequences of positive integers $\{l(k)\}$ and $\{m(k)\}$ such that for all positive integers $k$,

$$
\begin{gathered}
m(k)>l(k)>k \\
\max \left\{d\left(g x_{l(k)}^{1}, g x_{m(k)}^{1}\right), d\left(g x_{l(k)}^{2}, g x_{m(k)}^{2}\right), \cdots, d\left(g x_{l(k)}^{r}, g x_{m(k)}^{r}\right)\right\} \geq \varepsilon
\end{gathered}
$$

and

$$
\max \left\{d\left(g x_{l(k)}^{1}, g x_{m(k)-1}^{1}\right), d\left(g x_{l(k)}^{2}, g x_{m(k)-1}^{2}\right), \ldots, d\left(g x_{l(k)}^{r}, g x_{m(k)-1}^{r}\right)\right\}<\varepsilon
$$

Now,

$$
\begin{aligned}
d\left(g x_{l(k)}^{1}, g x_{m(k)}^{1}\right) & =d\left(F\left(x_{l(k)-1}^{1}, x_{l(k)-1}^{2}, \cdots, x_{l(k)-1}^{r}\right), F\left(x_{m(k)-1}^{1}, x_{m(k)-1}^{2}, \ldots, x_{m(k)-1}^{r}\right)\right) \\
& \leq \varphi\left(\max \left\{d\left(g x_{l(k)-1}^{n}, g x_{m(k)-1}^{n}\right)\right\}\right), n=1,2, \cdots, r .
\end{aligned}
$$

Similarly, $d\left(g x_{l(k)}^{2}, g x_{m(k)}^{2}\right) \leq \varphi\left(\max \left\{d\left(g x_{l(k)-1}^{n}, g x_{m(k)-1}^{n}\right)\right\}\right), \quad n=1,2, \cdots, r$ $\vdots$

$$
d\left(g x_{l(k)}^{r}, g x_{m(k)}^{r}\right) \leq \varphi\left(\max \left\{d\left(g x_{l(k)-1}^{n}, g x_{m(k)-1}^{n}\right)\right\}\right), \quad n=1,2, \cdots, r
$$

Thus,

$$
\begin{aligned}
\varepsilon & \leq \max \left\{d\left(g x_{l(k)}^{1}, g x_{m(k)}^{1}\right), d\left(g x_{l(k)}^{2}, g x_{m(k)}^{2}\right), \cdots, d\left(g x_{l(k)}^{r}, g x_{m(k)}^{r}\right)\right\} \\
& \leq \varphi\left(\max \left\{d\left(g x_{l(k)-1}^{n}, g x_{m(k)-1}^{n}\right)\right\}\right), n=1,2, \cdots, r
\end{aligned}
$$

Again, the triangular inequality and (3.17) gives

$$
\begin{aligned}
d\left(g x_{l(k)-1}^{1}, g x_{m(k)-1}^{1}\right) & \leq d\left(g x_{l(k)-1}^{1}, g x_{l(k)}^{1}\right)+d\left(g x_{l(k)}^{1}, g x_{m(k)-1}^{1}\right) \\
& \leq d\left(g x_{l(k)-1}^{1}, g x_{l(k)}^{1}\right)+\varepsilon, \quad n=1,2, \cdots, r
\end{aligned}
$$

and

$$
d\left(g x_{l(k)-1}^{2}, g x_{m(k)-1}^{2}\right) \leq d\left(g x_{l(k)-1}^{n}, g x_{l(k)}^{n}\right)+\varepsilon, n=1,2, \cdots, r
$$

$$
d\left(g x_{l(k)-1}^{r}, g x_{m(k)-1}^{r}\right) \leq d\left(g x_{l(k)-1}^{n}, g x_{l(k)}^{n}\right)+\varepsilon, n=1,2, \cdots, r .
$$

i.e., we have

$$
\begin{aligned}
& \max \left\{d\left(g x_{l(k)-1}^{1}, g x_{m(k)-1}^{1}\right), d\left(g x_{l(k)-1}^{2}, g x_{m(k)-1}^{2}\right), \cdots, d\left(g x_{l(k)-1}^{r}, g x_{m(k)-1}^{r}\right)\right\} \\
& \leq \max \left\{\leq d\left(g x_{l(k)-1}^{n}, g x_{l(k)}^{n}\right)\right\}+\varepsilon, n=1,2, \cdots, r
\end{aligned}
$$

Also,

$$
\begin{gathered}
d\left(g x_{m(k)}^{1}, g x_{l(k)}^{1}\right) \leq d\left(g x_{m(k)}^{1}, g x_{m(k)-1}^{1}\right)+d\left(g x_{m(k)-1}^{1}, g x_{l(k)-1}^{1}\right)+d\left(g x_{l(k)-1}^{1}, g x_{l(k)}^{1}\right) \\
d\left(g x_{m(k)}^{2}, g x_{l(k)}^{2}\right) \leq d\left(g x_{m(k)}^{2}, g x_{m(k)-1}^{2}\right)+d\left(g x_{m(k)-1}^{2}, g x_{l(k)-1}^{2}\right)+d\left(g x_{l(k)-1}^{2}, g x_{l(k)}^{2}\right)
\end{gathered}
$$


$\vdots$

$$
d\left(g x_{m(k)}^{r}, g x_{l(k)}^{r}\right) \leq d\left(g x_{m(k)}^{r}, g x_{m(k)-1}^{r}\right)+d\left(g x_{m(k)-1}^{r}, g x_{l(k)-1}^{r}\right)+d\left(g x_{l(k)-1}^{r}, g x_{l(k)}^{r}\right)
$$

Using (3.17), (3.19) and (3.22), we have

$$
\begin{aligned}
& \varepsilon \leq \max \left\{d\left(g x_{l(k)}^{1}, g x_{m(k)}^{1}\right), d\left(g x_{l(k)}^{2}, g x_{m(k)}^{2}\right), \cdots, d\left(g x_{l(k)}^{r}, g x_{m(k)}^{r}\right)\right\} \\
& \leq \max \left\{d\left(g x_{m(k)}^{1}, g x_{m(k)-1}^{1}\right), d\left(g x_{m(k)}^{2}, g x_{m(k)-1}^{2}\right), \cdots, d\left(g x_{m(k)}^{r}, g x_{m(k)-1}^{r}\right)\right\} \\
& +\max \left\{d\left(g x_{m(k)-1}^{1}, g x_{l(k)-1}^{1}\right), d\left(g x_{m(k)-1}^{2}, g x_{l(k)-1}^{2}\right), \cdots, d\left(g x_{m(k)-1}^{r}, g x_{l(k)-1}^{r}\right)\right\} \\
& +\max \left\{d\left(g x_{l(k)-1}^{1}, g x_{l(k)}^{1}\right), d\left(g x_{l(k)-1}^{2}, g x_{l(k)}^{2}\right), \cdots, d\left(g x_{l(k)-1}^{r}, g x_{l(k)}^{r}\right)\right\} .
\end{aligned}
$$

Letting $k \rightarrow \infty$ in above equation, we get

$$
\lim _{k \rightarrow \infty}\left(\max \left\{d\left(g x_{l(k)-1}^{1}, g x_{m(k)-1}^{1}\right), d\left(g x_{l(k)-1}^{2}, g x_{m(k)-1}^{2}\right), \cdots, d\left(g x_{l(k)-1}^{r}, g x_{m(k)-1}^{r}\right)\right\}\right)=\varepsilon
$$

Finally, letting $k \rightarrow \infty$ in (3.17) and using (3.19) and (3.23), we get

$$
\varepsilon \leq \max \left\{d\left(g x_{l(k)}^{1}, g x_{m(k)}^{1}\right), d\left(g x_{l(k)}^{2}, g x_{m(k)}^{2}\right), \cdots, d\left(g x_{l(k)}^{r}, g x_{m(k)}^{r}\right)\right\} \leq \varphi(\varepsilon)<\varepsilon
$$

which is a contradiction. Therefore, $\left\{g\left(x_{m}^{1}\right)\right\},\left\{g\left(x_{m}^{2}\right)\right\},\left\{g\left(x_{m}^{3}\right)\right\}, \cdots,\left\{g\left(x_{m}^{r}\right)\right\}$ are Cauchy sequences. Since the metric space $(X, d)$ is complete, so there exist $x^{1}, x^{2}, \cdots, x^{r} \in X$ such that

$$
\lim _{m \rightarrow \infty} g\left(x_{m}^{1}\right)=x^{1}, \lim _{m \rightarrow \infty} g\left(x_{m}^{2}\right)=x^{2}, \cdots, \lim _{m \rightarrow \infty} g\left(x_{m}^{r}\right)=x^{r} .
$$

As $g$ is continuous, so from (2.26), we have

$$
\lim _{m \rightarrow \infty} g\left(g\left(x_{m}^{1}\right)\right)=g\left(x^{1}\right), \lim _{m \rightarrow \infty} g\left(g\left(x_{m}^{2}\right)\right)=g\left(x^{2}\right), \cdots, \lim _{m \rightarrow \infty} g\left(g\left(x_{m}^{r}\right)\right)=g\left(x^{r}\right)
$$

By the compatibility of $g$ and $F$, we have

$$
\begin{aligned}
& \lim _{n \rightarrow \infty} d\left(g\left(F\left(x_{m}^{1}, x_{m}^{2}, \cdots, x_{m}^{r}\right)\right), F\left(g\left(x_{m}^{1}\right), g\left(x_{m}^{2}\right), \cdots, g\left(x_{m}^{r}\right)\right)\right)=0, \\
& \lim _{n \rightarrow \infty} d\left(g\left(F\left(x_{m}^{2}, \cdots, x_{m}^{r}, x_{m}^{1}\right)\right), F\left(g\left(x_{m}^{2}\right), \cdots, g\left(x_{m}^{r}\right), g\left(x_{m}^{1}\right)\right)\right)=0, \\
& \lim _{n \rightarrow \infty} d\left(g\left(\left(F\left(x_{m}^{r}, x_{m}^{1}, \cdots, x_{m}^{r-1}\right)\right)\right), F\left(g\left(x_{m}^{r}\right), g\left(x_{m}^{1}\right), \cdots, g\left(x_{m}^{r-1}\right)\right)\right)=0 .
\end{aligned}
$$

Now, we show that $F$ and $g$ have an r-tupled coincidence point. To accomplish this, suppose (a) holds. i.e. $F$ is continuous, then using (3.28) and (3.8), we see that

$$
\begin{aligned}
& d\left(g\left(x^{1}\right), F\left(x^{1}, x^{2}, \cdots, x^{r}\right)\right) \\
& =\lim _{n \rightarrow \infty} d\left(g\left(g\left(x_{m+1}^{1}\right)\right), F\left(g\left(x_{m}^{1}\right), g\left(x_{m}^{2}\right), \cdots, g\left(x_{m}^{r}\right)\right)\right) \\
& =\lim _{n \rightarrow \infty} d\left(g\left(F\left(x_{m}^{1}, x_{m}^{2}, \cdots, x_{m}^{r}\right)\right), F\left(g\left(x_{m}^{1}\right), g\left(x_{m}^{2}\right), \cdots, g\left(x_{m}^{r}\right)\right)\right)=0,
\end{aligned}
$$

which gives $g\left(x^{1}\right)=F\left(x^{1}, x^{2}, \cdots, x^{r}\right)$. Similarly, we can prove $g\left(x^{2}\right)=F\left(x^{2}, \cdots, x^{r}, x^{1}\right), \cdots$,

$$
g\left(x^{r}\right)=F\left(x^{r}, x^{1}, \cdots, x^{r-1}\right)
$$


Hence $\left(x^{1}, x^{2}, \cdots, x^{r}\right) \in \prod_{i=1}^{r} X^{i}$ is an r-tupled coincidence point of the maps $F$ and $g$.

If (b) holds, since $g\left(x_{m}^{i}\right)$ is non-decreasing or non-increasing as $i$ is odd or even and $g\left(x_{m}^{i}\right) \rightarrow x^{i}$ as $m \rightarrow \infty$, we have $g\left(x_{m}^{i}\right) \preceq x^{i}$, when $i$ is odd while $g\left(x_{m}^{i}\right) \succeq x^{i}$, when $i$ is even. Since $g$ is monotonically increasing, therefore

$$
g\left(g\left(x_{m}^{i}\right)\right) \preceq g\left(x^{i}\right) \text { when } i \text { is odd, }
$$

$g\left(g\left(x_{m}^{i}\right)\right) \succeq g\left(x^{i}\right)$ when $i$ is even.

Now, using triangle inequality together with (3.8), we get

$$
\begin{aligned}
& d\left(g\left(x^{1}\right), F\left(x^{1}, x^{2}, \cdots, x^{r}\right)\right) \\
& \leq d\left(g\left(x^{1}\right), g\left(x_{m+1}^{1}\right)\right)+d\left(g\left(x_{m+1}^{1}\right), F\left(x^{1}, x^{2}, \cdots, x^{r}\right)\right) \\
& \leq d\left(g\left(x^{1}\right), g\left(x_{m+1}^{1}\right)\right)+d\left(g\left(F\left(x_{m}^{1}, x_{m}^{2}, \cdots, x_{m}^{r}\right)\right), F\left(g\left(x_{m}^{1}\right), g\left(x_{m}^{2}\right), \cdots, g\left(x_{m}^{r}\right)\right)\right) \\
& \rightarrow 0 \text { as } n \rightarrow \infty .
\end{aligned}
$$

Therefore, $g\left(x^{1}\right)=F\left(x^{1}, x^{2}, \cdots, x^{r}\right)$. Similarly we can prove

$$
g\left(x^{2}\right)=F\left(x^{2}, \cdots, x^{r}, x^{1}\right), \cdots, \quad g\left(x^{r}\right)=F\left(x^{r}, x^{1}, \cdots, x^{r-1}\right)
$$

Thus the theorem follows.

Corollary 3.1 Under the hypothesis of theorem 3.2 and satisfying contractive condition as (3.31) $d\left(F\left(x^{1}, x^{2}, \cdots, x^{r}\right), F\left(y^{1}, y^{2}, \cdots, y^{r}\right)\right) \leq \frac{k}{r} \sum_{n=1}^{r} d\left(g\left(x^{n}\right), g\left(y^{n}\right)\right), k \in[0,1)$.

Then $F$ and $g$ have a r-tupled coincidence point.

Proof: If we put $\varphi(t)=k \cdot t$ with $k \in[0,1)$ in theorem 3.2, we get the corollary.

\section{Uniqueness of r-tupled fixed point}

For all $\left(x^{1}, x^{2}, \cdots, x^{r}\right),\left(y^{1}, y^{2}, \cdots, y^{r}\right) \in X^{r}$,

$$
\begin{aligned}
& \left(x^{1}, x^{2}, \cdots, x^{r}\right) \preceq\left(y^{1}, y^{2}, \cdots, y^{r}\right) \\
& \Leftrightarrow x^{1} \preceq y^{1}, x^{2} \succeq y^{2}, \cdots, x^{r} \succeq y^{r} .
\end{aligned}
$$

We say that $\left(x^{1}, x^{2}, \cdots, x^{r}\right)=\left(y^{1}, y^{2}, \cdots, y^{r}\right) \Leftrightarrow x^{1}=y^{1}, x^{2}=y^{2}, \cdots, x^{r}=y^{r}$.

Theorem 3.3 In addition to the hypothesis of theorem 3.1, suppose that for every

$$
\left(x^{1}, x^{2}, \cdots, x^{r}\right),\left(y^{1}, y^{2}, \cdots, y^{r}\right) \in X^{r}
$$

Then exist $\left(z^{1}, z^{2}, \cdots, z^{r}\right) \in X^{r}$ such that $F\left(\left(z^{1}, z^{2}, \cdots, z^{r}\right),\left(z^{2}, z^{3}, \cdots, z^{r}, z^{1}\right), \cdots,\left(z^{r}, z^{1}, \cdots, z^{r-1}\right)\right)$ is comparable to

$$
F\left(\left(x^{1}, x^{2}, \cdots, x^{r}\right), F\left(x^{2}, x^{3}, \cdots, x^{r}, x^{1}\right), \cdots, F\left(x^{r}, x^{1}, \cdots, x^{r-1}\right)\right)
$$

And

$$
F\left(\left(y^{1}, y^{2}, \cdots, y^{r}\right), F\left(y^{2}, y^{3}, \cdots, y^{r}, y^{1}\right), \cdots, F\left(y^{r}, y^{1}, \cdots, y^{r-1}\right)\right)
$$

Then $F$ and $g$ have a unique r-coincidence point, which is a fixed point of $g: X \rightarrow X$ and $F: \prod_{i=1}^{r} X^{i} \rightarrow X$. That is there exists a unique $\left(x^{1}, x^{2}, \cdots, x^{r}\right) \in X^{r}$ such that 


$$
x^{i}=g\left(x^{i}\right)=F\left(x^{i}, x^{1}, \cdots, x^{i-1}\right) \text { for all } i \in\{1,2, \cdots, r\}
$$

Proof. By theorem 3.2, the set of r-coincidence points is non-empty. Now, suppose that $\left(x^{1}, x^{2}, \cdots, x^{r}\right)$ and $\left(y^{1}, y^{2}, \cdots, y^{r}\right)$ are two coincidence points of $F$ and $g$, that is $g\left(x^{i}\right)=F\left(x^{i}, x^{1}, \cdots, x^{i-1}\right)$ for all $i \in\{1,2, \cdots, r\}$ and $g\left(y^{i}\right)=F\left(y^{i}, y^{1}, \cdots, y^{i-1}\right)$ for all $i \in\{1,2, \cdots, r\}$.

We will show that $g\left(x^{i}\right)=g\left(y^{i}\right)$ for all $i \in\{1,2, \cdots, r\}$.

By assumption, there exists $\left(z^{1}, z^{2}, \cdots, z^{r}\right) \in X^{r}$ such that

$$
F\left(\left(z^{1}, z^{2}, \cdots, z^{r}\right), F\left(z^{2}, z^{3}, \cdots, z^{r}, z^{1}\right), \cdots, F\left(z^{r}, z^{1}, \cdots, z^{r-1}\right)\right)
$$

is comparable to

$$
F\left(\left(x^{1}, x^{2}, \cdots, x^{r}\right), F\left(x^{2}, x^{3}, \cdots, x^{r}, x^{1}\right), \cdots, F\left(x^{r}, x^{1}, \cdots, x^{r-1}\right)\right)
$$

and

$$
F\left(\left(y^{1}, y^{2}, \cdots, y^{r}\right), F\left(y^{2}, y^{3}, \cdots, y^{r}, y^{1}\right), \cdots, F\left(y^{r}, y^{1}, \cdots, y^{r-1}\right)\right) .
$$

Let $z_{0}^{i}=z^{i}$ for all $i \in\{1,2, \cdots, r\}$. Since $F\left(X^{r}\right) \subseteq g(X)$, we can choose $z_{1}^{i} \in X$ such that $g\left(z_{1}^{i}\right)=F\left(z_{0}^{i}, z_{0}^{1}, \cdots, z_{0}^{i-1}\right)$ for all $i \in\{1,2, \cdots, r\}$. By a similar reason, we can inductively define sequences $\left\{g\left(z_{n}^{i}\right)\right\}, n \in N$ for all $i \in\{1,2, \cdots, r\}$ such that $g\left(z_{n+1}^{i}\right)=F\left(z_{n}^{i}, z_{n}^{1}, \cdots, z_{n}^{i-1}\right)$ for all $i \in\{1,2, \cdots, r\}$.

In addition, let $x_{0}^{i}=x^{i}$ and $y_{0}^{i}=y^{i}$ for all $i \in\{1,2, \cdots, r\}$ and in the same way, define the sequences $\left\{g\left(x_{n}^{i}\right)\right\}$ and $\left\{g\left(y_{n}^{i}\right)\right\}, n \in N$ for all $i \in\{1,2, \cdots, r\}$. Since

$$
F\left(x^{1}, x^{2}, \cdots, x^{r}\right), F\left(x^{2}, x^{3}, \cdots, x^{r}, x^{1}\right), \cdots, F\left(x^{r}, x^{1}, \cdots, x^{r-1}\right)=\left(g x_{1}^{1}, g x_{1}^{2}, \cdots, g x_{1}^{r}\right)
$$

And

$$
F\left(z^{1}, z^{2}, \cdots, z^{r}\right), F\left(z^{2}, z^{3}, \cdots, z^{r}, z^{1}\right), \cdots, F\left(z^{r}, z^{1}, \cdots, z^{r-1}\right)=\left(g z_{1}^{1}, g z_{1}^{2}, \cdots, g z_{1}^{r}\right)
$$

are comparable, then

$g\left(x_{1}^{i}\right) \leq g\left(z_{1}^{i}\right)$ for all $i \in\{1,2, \cdots, r\}$ if $i$ is odd,

$g\left(x_{1}^{i}\right) \geq g\left(z_{1}^{i}\right)$ for all $i \in\{1,2, \cdots, r\}$ if $i$ is even.

We have

$$
\begin{gathered}
g\left(x^{2 i-1}\right)=g\left(x_{1}^{2 i-1}\right) \leq g\left(z_{2}^{2 i-1}\right) \leq \cdots \leq g\left(z_{r}^{2 i-1}\right), \\
g\left(x^{2 i}\right)=g\left(x_{1}^{2 i}\right) \leq g\left(z_{2}^{2 i}\right) \leq \cdots \leq g\left(z_{r}^{2 i}\right) .
\end{gathered}
$$

Then $\left(g\left(x^{1}\right), g\left(x^{2}\right), \cdots, g\left(x^{r}\right)\right)$ and $\left(g\left(z_{n}^{1}\right), g\left(z_{n}^{2}\right), \cdots, g\left(z_{n}^{r}\right)\right)$ are comparable for all $n \in N$. It follows from condition (3.4) of theorem 3.2

$$
\begin{aligned}
d\left(g\left(x^{i}\right), g\left(z_{n+1}^{i}\right)\right) & =d\left(F\left(x^{i}, x^{1}, \cdots, x^{i-1}\right), F\left(z_{n}^{i}, z_{n}^{1}, \cdots, z_{n}^{i-1}\right)\right) \\
& \leq \varphi\left(\frac{1}{r} \sum_{i=1}^{r} d\left(g x^{i}, g z_{n}^{i}\right)\right), \text { for all } i \in\{1,2, \cdots, r\} .
\end{aligned}
$$

Summing, we get 


$$
\begin{aligned}
& \frac{1}{r}\left[d\left(g\left(x^{1}\right), g\left(z_{n}^{1}\right)\right)+d\left(g\left(x^{2}\right), g\left(z_{n}^{2}\right)\right)+\cdots+d\left(g\left(x^{r}\right), g\left(z_{n}^{r}\right)\right)\right] \\
& \leq \frac{r}{r} \varphi\left(\frac{d\left(g\left(x^{1}\right), g\left(z_{n}^{1}\right)\right)+d\left(g\left(x^{2}\right), g\left(z_{n}^{2}\right)\right)+\cdots+d\left(g\left(x^{r}\right), g\left(z_{n}^{r}\right)\right)}{r}\right)
\end{aligned}
$$

It follows that

$$
\begin{aligned}
& \frac{1}{r}\left[d\left(g\left(x^{1}\right), g\left(z_{n+1}^{1}\right)\right)+d\left(g\left(x^{2}\right), g\left(z_{n+1}^{2}\right)\right)+\cdots+d\left(g\left(x^{r}\right), g\left(z_{n+1}^{r}\right)\right)\right] \\
& \leq \varphi^{n}\left(\frac{d\left(g\left(x^{1}\right), g\left(z^{1}\right)\right)+d\left(g\left(x^{2}\right), g\left(z^{2}\right)\right)+\cdots+d\left(g\left(x^{r}\right), g\left(z^{r}\right)\right)}{r}\right)
\end{aligned}
$$

For all $n \geq 1$. Note that $\varphi(0)=0, \varphi(t)<t, \lim _{r \rightarrow t^{+}} \varphi(r)<t$ for $t>0$ imply that $\lim _{n \rightarrow \infty} \varphi^{n}(t)=0$ for all $t>0$. Hence from (3.32) we have

$$
\lim _{n \rightarrow \infty} d\left(g\left(x^{i}\right), g\left(z_{n+1}^{i}\right)\right)=0 \text { for all } i \in\{1,2, \cdots, r\} .
$$

Similarly, one can prove that

$$
\lim _{n \rightarrow \infty} d\left(g\left(y^{i}\right), g\left(z_{n+1}^{i}\right)\right)=0 \text { for all } i \in\{1,2, \cdots, r\} .
$$

Using (3.34), (3.35) and triangle inequality we get

$$
d\left(g\left(x^{i}\right), g\left(y^{i}\right)\right) \leq d\left(g\left(x^{i}\right), g\left(z_{n+1}^{i}\right)\right)+d\left(g\left(z_{n+1}^{i}\right), g\left(y^{i}\right)\right) \rightarrow 0,
$$

As $n \rightarrow \infty$ for all $i \in\{1,2, \cdots, r\}$. Hence, $g\left(x^{i}\right)=g\left(y^{i}\right)$, therefore (3.32) is proved.

Since $g\left(x^{i}\right)=F\left(x^{i}, x^{1}, \cdots, x^{i-1}\right)$ for all $i \in\{1,2, \cdots, r\}$, by the commutativity of $F$ and $g$, we have

$$
g\left(g\left(x^{i}\right)\right)=g\left(F\left(x^{i}, x^{1}, \cdots, x^{i-1}\right)\right)=F\left(g x^{i}, g x^{1}, \cdots, g x^{i-1}\right)
$$

Denote $g x^{i}=u^{i}$ for all $i \in\{1,2, \cdots, r\}$. From (3.36), we have

$$
g\left(u^{i}\right)=g\left(g x^{i}\right)=F\left(u^{i}, u^{1}, \cdots, u^{i-1}\right) \text { for all } i \in\{1,2, \cdots, r\} .
$$

Hence $\left(u^{i}, u^{1}, \cdots, u^{i-1}\right)$ is a r-coincidence point of $F$ and $g$.

It follows from (3.32) $y^{i}=u^{i}$ and so

$$
g\left(y^{i}\right)=g\left(u^{i}\right) \text { for all } i \in\{1,2, \cdots, r\} .
$$

This means that

$$
g\left(u^{i}\right)=u^{i} \text { for all } i \in\{1,2, \cdots, r\}
$$

Now, from (3.37), we have

$$
u^{i}=g\left(u^{i}\right)=F\left(u^{i}, u^{1}, \cdots, u^{i-1}\right) \text { for all } i \in\{1,2, \cdots, r\} .
$$

Hence, $\left(u^{1}, u^{2}, \cdots, u^{r}\right)$ is a r-fixed point of $F$ and a fixed point of $g$.

To prove the uniqueness of the fixed point, assume that $\left(v^{1}, v^{2}, \cdots, v^{r}\right)$ is another r-fixed point. Then by 
(3.32) we have

$$
u^{i}=g\left(u^{i}\right)=v^{i}=g\left(v^{i}\right) \text { for all } i \in\{1,2, \cdots, r\}
$$

Thus, $\left(u^{1}, u^{2}, \cdots, u^{r}\right)=\left(v^{1}, v^{2}, \cdots, v^{r}\right)$. This completes the proof.

\section{Acknowledgements}

Authors are highly thankful for the financial support of this paper to Deanship of Scientific Research, Jazan University, K.S.A.

\section{Conflict of Interest}

Authors declare that they have no conflict of interest.

\section{References}

[1] Choudhary, B.S. and Kundu, A. (2010) Coupled Coincidence Point Results in Ordered Metric Spaces for Compatible Mappings. Nonlinear Analysis: Theory, Method and Applications A, 73, 2524-2531. http://dx.doi.org/10.1016/j.na.2010.06.025

[2] Choudhary, B.S., Das K. and Das, P. Coupled Coincidence Point Theorems in Partially Ordered Fuzzy Metric Spaces, Fuzzy Sets and System ( in Press).

[3] Bhaskar, T.G. and Lakshmikantham, V. (2006) Fixed Point Theorems in Partially Ordered Metric Spaces and Applications. Nonlinear Analysis: Theory, Methods and Applications, 65, 1379-1393. http://dx.doi.org/10.1016/j.na.2005.10.017

[4] Lakshmikantham, V. and Ciric, L. (2009) Coupled Fixed Point Theorems for Nonlinear Contractions in Partially Ordered Metric Spaces. Nonlinear Analysis: Theory, Method and Applications, 70, 4341-4349. http://dx.doi.org/10.1016/j.na.2008.09.020

[5] Alotaibi, A. and Alsulami, S. (2011) Coupled Coincidence Points for Monotone Operators in Partially Ordered Metric Spaces. Fixed Point Theory and Applications, 2011, 44. http://dx.doi.org/10.1186/1687-1812-2011-44

[6] Ran, A.C.M. and Reuring, M.C.B. (2004) A Fixed Point Theorem in Partially Metric Spaces and Some Applications in Partially Ordered Metric Spaces. Proceedings of the American Mathematical Society, 132, 1435-1443. http://dx.doi.org/10.1090/S0002-9939-03-07220-4

[7] Karapinar, E., Luong, N.V. and Thuan, N.X. (2012) Coupled Coincidence Points for Mixed Monotone Operators in Partially Ordered Metric Spaces. Arabian Journal of Mathematics, 1, 329-339. http://dx.doi.org/10.1007/s40065-012-0027-0

[8] Karapinar, E. and Irhan, I.M. (1999) Fixed Point Theorems for Operators on Partial Metric Spaces. Appl. Categ, Structures, 7, 71-83. http://dx.doi.org/10.1023/A:1008684018933

[9] Jungck, G. (1986) Compatible Mappings and Common Fixed Points. International Journal of Mathematics and Mathematical Sciences, 9, 771-779. http://dx.doi.org/10.1155/S0161171286000935

[10] Aydi, H. (2011) Some Coupled Fixed Point Results on Partial Metric Spaces. International Journal of Mathematics and Mathematical Sciences, 2011, Article ID: 647091, $11 \mathrm{p}$.

[11] Nieto, J.J. and Rodriguez-Lopez, R. (2005) Contractive Mapping Theorems in Partially Ordered Sets and Applications to Ordinary Differential Equation. Order, 22, 223-239. http://dx.doi.org/10.1007/s11083-005-9018-5

[12] Sumitra, D., Manro, S., Bhatia, S.S., Kumar, S. and Kumum, P. (2013) Weakly Compatibly Mapping with CLR Mapping in Fuzzy Metric Saces. Journal Nonlinear Analysis and Applications, 2013, 1-12. http://dx.doi.org/10.5899/2013/jnaa-00206

[13] Gugani, M., Agarwal, M. and Chugh, R. (2012) Common Fixed Point Results in G-Metric Spaces and Applications. International Journal of Computer Applications, 43, No. 11.

[14] Nguyen, V. and Nguyen, X. (2010) Coupled Fixed Point Theorems in Partially Ordered Metric Spaces. Bulletin of Mathematical Analysis and Applications, 2, 16-24.

[15] Berinde, V. and Borcut, M. (2011) Tripled Fixed Point Theorems for Contractive Type Mappings in Partially Ordered Metric Spaces. Nonlinear Analysis, 74, 4889-4897. http://dx.doi.org/10.1016/j.na.2011.03.032

[16] Karpinar, E. (2012) Quartet Fixed Point for Nonlinear Contraction. http://arxiv.org/abs/1106.5472 
[17] Sumitra, D., Chaun, S. and Pant, B.D. (2013) Coincidence and Common Fixed Point Theorems in Intutionistic Fuzzy -Metric Spaces. Far East Journal of Mathematical Science, 79, 25-48.

[18] Sumitra, D., Chauhan, S. and Kadelburg, Z. (2013) A Common Fixed Point Theorem in Metric Space under General Contractive Condition. Journal of Applied Mathematics, 2013, Article ID: 510691, 7 p.

[19] Sumitraand Manro, S. (2014) Coincidence and Common Fixed Point of Weakly Compatible Maps in Fuzzy Metric Spaces. Applied Mathematics, 5, 1335-1348.

[20] Sumitra, D., Imdad, M. and Chauhan, S. (2013) Unified Fixed Point Theorems via Common Limit Range Property in Modified Intuitionistic Fuzzy Metric Spaces. Abstract and Applied Analysis, 2013, Article ID: 413473, 11 p.

[21] Shatanawi, W. (2011) Coupled Fixed Point Theorems in Generalized Metric Spaces. Hacettepe Journal of Mathematics and Statistics, 40, 441-447.

[22] Imdad, M., Soliman, A.H., Choudhary, B.S. and Das, P. (2013) On n-Tupled Coincidence Point Results in Metric Spaces. Journal of Operators, 2013, Article ID: 532867, 8 p.

[23] Jungck, G. (1976) Commuting Mappings and Fixed Point. American Mathematical Monthly, 83, 261-263. http://dx.doi.org/10.2307/2318216 